\title{
Fuzzy Nonparametric DTI Segmentation for Robust Cingulum-Tract Extraction
}

\author{
Suyash P. Awate, Hui Zhang, and James C. Gee \\ Department of Radiology, University of Pennsylvania, Philadelphia, PA 19104, USA \\ \{awate, gee\}@mail.med.upenn.edu, huiz@seas. upenn.edu
}

\begin{abstract}
This paper presents a novel segmentation-based approach for fiber-tract extraction in diffusion-tensor (DT) images. Typical tractography methods, incorporating thresholds on fractional anisotropy and fiber curvature to terminate tracking, can face serious problems arising from partial voluming and noise. For these reasons, tractography often fails to extract thin tracts with sharp changes in orientation, e.g. the cingulum. Unlike tractography - which disregards the information in the tensors that were previously tracked - the proposed method extracts the cingulum by exploiting the statistical coherence of tensors in the entire structure. Moreover, the proposed segmentation-based method allows fuzzy class memberships to optimally extract information within partial-volumed voxels. Unlike typical fuzzy-segmentation schemes employing Gaussian models that are biased towards ellipsoidal clusters, the proposed method models the manifolds underlying the classes by incorporating nonparametric data-driven statistical models. Furthermore, it exploits the nonparametric model to capture the spatial continuity and structure of the fiber bundle. The results on real DT images demonstrate that the proposed method extracts the cingulum bundle significantly more accurately as compared to tractography.
\end{abstract}

\section{Introduction}

Diffusion tensor (DT) magnetic resonance (MR) imaging allows us to differentiate the cerebral white/gray-matter structures such as the corpus callosum, thalamic nuclei, cingulum, etc. Extraction of these structures is of key interest in clinical studies concerning schizophrenia, Parkinson's disease, and Alzheimer's disease [ $\left.1 \begin{array}{ll|l|l|l} & 2 & 3 & 4 & 5\end{array}\right]$. The driving clinical application for this paper is to investigate whether there are changes in the anterior cingulum in cocaine users that are associated with decreased gray-matter concentration in the same region [6].

The efficacy of tract-extraction methods on DT images can be severely limited by noise and partial-voluming artifacts. For instance, tractography methods often fails to extract the cingulum 34 - a thin tract that undergoes sharp changes in orientation and severe partial-volume contamination from the highlyanisotropic corpus callosum and the highly-isotropic ventricles lying adjacent to it (Figure 11). Tractography methods, which incorporate thresholds on the fractional anisotropy (FA) and fiber curvature to terminate tracking, can also consistently underestimate the size of fiber bundles [7].

N. Ayache, S. Ourselin, A. Maeder (Eds.): MICCAI 2007, Part I, LNCS 4791, pp. 294-301, 2007.

(C) Springer-Verlag Berlin Heidelberg 2007 
Alternative approaches for extracting structures from DT images rely on segmentation approaches. Unlike tractography that disregards the information in the tensors that were previously tracked, segmentation approaches exploit the coherence of tensors in the entire structure of interest. Moreover, segmentation methods that allow fuzzy class memberships optimally extract information within partial-volumed voxels - crisp segmentations incorrectly account for this information towards the representation of a single class. In these ways, segmentation schemes can be more robust to DT-image artifacts.

Previous work in DTI segmentation employs Gaussian models that may not effectively model the tensor statistics because they are inherently biased towards ellipsoidal clusters. Fundamental anatomical characteristics of fiber bundles make fibers change their orientation significantly, e.g. cingulum (Figure 10), as they connect different structures. Thus, tensors in fiber bundles inherently lie on manifolds that do not conform to Gaussian models that are characterized by the mean. For instance, tensors in U-shaped/C-shaped bundles, that include tensors which start and end at similar orientations, must lie on a closed manifold.

This paper makes several contributions. It proposes a novel scheme based on fuzzy segmentation for extracting fiber bundles, e.g. cingulum, relying on a nonparametric statistical framework. The proposed method does not impose strong parametric tensor models, but adapts its models to arbitrary tensor probability density functions (PDFs), and the underlying manifolds, in data-driven ways. It extends the nonparametric model to capture the spatial continuity and structure of the fiber bundle. The fuzzy framework optimally extracts information from partial-volumed voxels. Results on real DT images show that the proposed method extracts the cingulum significantly more accurately than tractography.

\section{Related Work}

Typical methods for crisp and fuzzy segmentation 8910 rely on representing each class by only a single point in the feature space (the class mean) and measure class membership based on the Euclidean/Mahalanobis distance to the class mean. Such approaches, however, (a) bias the segmentation towards ellipsoidal clusters in feature space and (b) do not yield themselves easily in capturing the spatial structure of the fiber bundle in the image. Nonparametric modeling approaches for crisp structural-MR segmentation [11]2] address the concern in (a), but not in (b). The proposed method: (i) generalizes class representation in fuzzy methods to the manifold underlying the class, (ii) extends a data-driven nonparametric scheme to adaptively infer the spatial structure of the fiber bundle in the image, and (iii) extracts information from partial-volumed tensors in a principled manner by incorporating fuzzy class memberships.

Segmentation methods entail quantifying distances between tensors that respect the group structure of the Riemannian DT space [1314115. Lenglet et al. 1] use an affine-invariant Riemannian metric and model each class by a single Gaussian. Wang and Vemuri 2] use a piecewise-smooth Mumford-Shah framework to capture the spatial variations in tensors across a bundle. In contrast, 
the proposed method relies on nonparametric models and optimally extracts information from partial-volumed voxels using fuzzy class memberships.

The extraction of the cingulum tract, a key component of the limbic system that is relevant to memory and emotion, holds significance in many clinical studies relating to schizophrenia. Gong et al. 4. employ tractography to extract the cingulum bundle to study the left-right asymmetry in the structure. Their tractography results, however, clearly shows fibers "leaking" out of the cingulum. Concha et al. [3], acknowledging the impracticality of tractography for cingulum extraction, divide the cingulum into 3 parts (anterior, superior, descending) and perform tractography independently in each of these parts. In each part, they include tracts that pass through manually-selected regions-of-interest in the start, middle, and end of the part. The proposed method, on the other hand, extracts the cingulum in a simple, systematic, and optimal manner.

\section{Nonparametric Statistical Diffusion-Tensor Modeling}

This section describes the statistical formulation underlying the proposed nonparametric modeling technique. It starts by describing a generic kernel-based modeling scheme, that is independent of the particular metric associated with the Riemannian space. It then presents an appropriate tensor metric to considerably simplify the scheme, from a practical viewpoint, while maintaining the mathematical soundness of the framework.

We use kernel-based nonparametric PDF estimation based on a Parzen-window scheme in Riemannian space. For DT data, the kernel functions are smooth functions of the Riemannian geodesic distance on the tensor manifold. The mathematical expression for the Parzen-window tensor-PDF estimate is consistent with the expression of the usual (Euclidean) PDF estimate - it also relies on the intuitive notion of a kernel function having the largest value at the datum and monotonicallydecreasing values with increasing distance from the datum. In the Riemannian case, each datum is the intrinsic mean of the associated kernel for sufficiently small bandwidths. We now describe this scheme.

Let $\mathcal{M}$ be a compact Riemannian manifold without boundary, of dimension $D$, with an associated metric-tensor $g$. The metric tensor induces an inner product on the manifold that generates the geodesic distance function $d_{g}(\cdot, \cdot)$ between two entities on $\mathcal{M}$. Let $Z$ be a random variable on the probability space $(\Omega, \mathcal{A}, P)$ that takes values in $\mathcal{M}$. Let $\left\{z_{1}, z_{2}, \ldots, z_{n}\right\}$, where each $z_{i} \in \mathcal{M}$, be an independently-drawn and identically-distributed random sample derived from the $\operatorname{PDF} P(Z)$. Let $K(Z)$ be a nonnegative and sufficiently-smooth kernel function. Then, the consistent nonparametric PDF estimate is [16]:

$$
\hat{P}(z)=\frac{1}{N} \sum_{i=1}^{N} \frac{1}{\theta_{z_{i}}(z)} \frac{1}{\sigma^{D}} K\left(\frac{d_{g}\left(z, z_{i}\right)}{\sigma}\right),
$$

where $\sigma$ is the bandwidth associated with the kernel and $\theta_{a}(b)$ is the quotient of the canonical measure of the Riemannian metric $\exp _{a}^{*} g$ on $T_{a}(\mathcal{M})$ by the 
Lebesgue measure of the Euclidean structure $g_{a}$ on $T_{a}(\mathcal{M})$. We choose $K(\cdot)$ as the standard-Normal PDF: $K(\beta)=\left(1 /(2 \pi)^{D / 2}\right) \exp \left(-\beta^{2} / 2\right)$.

To evaluate the probability at any one point $z$ we need to, in general, compute $\theta_{z_{i}}(z)$ separately for all the points $z_{i}$ in the Parzen-window sample. This can become cumbersome, depending on the particular Riemannian tensor metric employed. The recently-proposed Log-Euclidean metric [14, in contrast to the affine-invariant Riemannian metric [13]1] zero curvature by mapping the DT space of $3 \times 3$ symmetric positive-definite matrices in an isomorphic, diffeomorphic, and isometric manner to the associated Euclidean vector space of $3 \times 3$ symmetric matrices. This mapping is precisely the matrix logarithm $\left(\right.$ denoted $\left.\log _{M}\right)$, i.e. $d_{g}\left(z, z_{i}\right)=\left\|\log _{M}(z)-\log _{M}\left(z_{i}\right)\right\|_{\text {Frobenius }}$ and $\theta_{z_{i}}(z)=1$. The Parzen-window PDF estimate then simplifies to $\hat{P}(z)=$ $(1 / N) \sum_{i=1}^{N} G\left(z ; z_{i}, \sigma\right)$, where $G\left(z ; z_{i}, \sigma\right)$ is a multidimensional isotropic Gaussian (mean $z_{i}$ and variance along each dimension $\sigma^{2}$ ) in the Riemannian space with $d_{g}(\cdot, \cdot)$ as the geodesic distance measure. Essentially, this scheme maps the diffusion tensors, using the matrix logarithm, to a Euclidean space and, in turn, computes probabilities using standard Parzen-window estimation in that space.

\section{Fuzzy Segmentation with Manifold-Based Models}

This section proposes a fuzzy-segmentation framework for fiber-tract extraction. It extracts tracts by iteratively optimizing an information-theoretic objective function and relying on nonparametric class models.

Our goal is to segment the image into $\mathcal{C}$ different classes $(c=1,2, \ldots, \mathcal{C})$. For extracting a single fiber bundle, $\mathcal{C}=2$. These classes are distinguished by their respective $\operatorname{PDFs}\left\{P_{c}(\cdot)\right\}_{c \in \mathcal{C}}$. The segmentation problem is, in a way, equivalent to that of deducing these PDFs.

Voxels in a fuzzy-segmentation framework can be members of more than one class - a standard notion in fuzzy set theory that does not constrain entities to belong to one set alone. We incorporate this notion using the fuzzy-membership functions that we define next. Consider $\mathcal{C}$ random variables $\left\{F_{c}: \mathcal{M} \rightarrow \Re\right\}_{c \in \mathcal{C}}$ that give a class-membership value for each element $\mathbf{z} \in \mathcal{M}$ belonging to class $c$. For all voxels $t$ and all classes $c$, we want $0 \leq F_{c}\left(\mathbf{z}_{t}\right) \leq 1$ and $\sum_{c=1}^{\mathcal{C}} F_{c}\left(\mathbf{z}_{t}\right)=1$. Under these constraints, we define the optimal fuzzy segmentation as:

$$
\underset{\left\{F_{c}(\cdot)\right\}_{c=1}^{\mathcal{C}}}{\operatorname{argmax}} \sum_{c=1}^{\mathcal{C}}\left(\int_{\mathcal{M}} F_{c}(\mathbf{z}) P_{c}(\mathbf{z}) \log P_{c}(\mathbf{z}) d \mathbf{z}-\alpha \int_{\mathcal{M}} F_{c}(\mathbf{z}) \log F_{c}(\mathbf{z}) d \mathbf{z}\right) .
$$

The first term of the energy in (2) is a modified Shannon's entropy that allows each observation $\mathbf{z} \in \mathcal{M}$ to contribute some amount to the entropy of every class $c$ that is proportional to its membership in class $c$ - partial-volumed voxels would contribute to the descriptions of multiple classes. $\alpha$ is a user-controlled parameter that controls the degree of fuzziness/softness imposed on the segmentation.

The method of Lagrange multipliers - and the short-hand terms $F_{c t}$ and $P_{c t}$ for $F_{c}\left(\mathbf{z}_{t}\right)$ and $P_{c}\left(\mathbf{z}_{t}\right)$, respectively - gives the objective function as: 


$$
\mathcal{J}=\sum_{c=1}^{C} \sum_{t \in \mathcal{T}}\left[F_{c t} \log P_{c t}-\alpha F_{c t} \log F_{c t}\right]+\sum_{t \in \mathcal{T}} \lambda_{t}\left[\sum_{c=1}^{C} F_{c t}-1\right],
$$

where $\left\{\lambda_{t}\right\}_{t \in \mathcal{T}}$ is the set of Lagrange multipliers.

The proposed method modifies the nonparametric statistical model in [16] so as to exploit the prior information that the tract is a spatially-continuous entity in the image-coordinate space and, subsequently, to infer the spatial structure of the tract. We do this by augmenting the tensor-valued feature space by the $3 \mathrm{D}$-coordinate space of the voxels. To include spatial information, we define the probabilities by $P_{c t}$ as: $P_{c t}=\left(1 /\left|\mathcal{S}_{c}\right|\right) \sum_{s \in \mathcal{S}_{c}} G\left(\mathbf{z}_{t} ; \mu_{c s}, \sigma_{c}\right) G\left(t ; \nu_{c s}, \rho_{c}\right)$, where the tensors $\left\{\mu_{c s}\right\}_{s \in \mathcal{S}_{c}}$, voxel locations $\left\{\nu_{c s}\right\}_{s \in \mathcal{S}_{c}}$, and bandwidths $\sigma_{c}$ and $\rho_{c}$ together model the PDF for class $c$ in the augmented feature space $\langle\mathcal{M}, \mathcal{T}\rangle$. This PDF captures: the manifold(s) underlying the tensor data in the tract, the variability of the tensors around the manifold(s), and the spatial continuity of the tract. For all classes, $\sigma_{c}$ is a penalized maximum-likelihood estimate for the Parzen-window PDF of the entire image $\mathbf{z}_{t \in \mathcal{T}}$ [17. We use $\rho_{c}=1$.

We need to maximize $\mathcal{J}$ with respect to $F_{c t}, \mu_{c s}, \nu_{c s}$, and $\lambda_{t}$. Solving the Karush-Kuhn-Tucker (KKT) necessary conditions for optimality gives the update for the fuzzy memberships $F_{c t}\left(\forall s \in \mathcal{S}_{c}, \forall c=1,2, \ldots, \mathcal{C}\right)$ as:

$$
F_{c t}=\frac{\left(P_{c t}\right)^{1 / \alpha}}{\sum_{c=1}^{\mathcal{C}}\left(P_{c t}\right)^{1 / \alpha}}
$$

Observe that, as expected, a large probability $P_{c t}$ for voxel $t$ to be in class $c$ produces a correspondingly larger membership value $F_{c t}$ of that voxel in class c. $\alpha \rightarrow \infty$ implies $F_{c t} \rightarrow 1 /|\mathcal{C}|$; a completely fuzzy segmentation. For $\alpha \rightarrow 0$, $F_{c t} \rightarrow 1$ if class $c$ with the largest $P_{c t}$; otherwise $F_{c t} \rightarrow 0$.

The updates for the class parameter $\mu_{c s}\left(\forall s \in \mathcal{S}_{c}, \forall c=1,2, \ldots, \mathcal{C}\right)$ are:

$$
\mu_{c s}=\frac{\sum_{t \in \mathcal{T}} F_{c t} \frac{G\left(\mathbf{z}_{t} ; \mu_{c s}, \sigma_{c}\right) G\left(t ; \nu_{c s}, \rho_{c}\right)}{P_{c t}} \mathbf{z}_{t}}{\sum_{t \in \mathcal{T}} F_{c t} \frac{G\left(\mathbf{z}_{t} ; \mu_{c s}, \sigma_{c}\right) G\left(t ; \nu_{c s}, \rho_{c}\right)}{P_{c t}}} ; \nu_{c s}=\frac{\sum_{t \in \mathcal{T}} F_{c t} \frac{G\left(\mathbf{z}_{t} ; \mu_{c s}, \sigma_{c}\right) G\left(t ; \nu_{c s}, \rho_{c}\right)}{P_{c t}} t}{\sum_{t \in \mathcal{T}} F_{c t} \frac{G\left(\mathbf{z}_{t} ; \mu_{c s}, \sigma_{c}\right) G\left(t ; \nu_{c s}, \rho_{c}\right)}{P_{c t}}}
$$

where the updated parameters $\mu_{c s}$ and $\nu_{c s}$ are weighted averages of the tensors $\mathbf{z}_{t}$ and voxel locations $t$, respectively. Observe that the weights take values between 0 and 1 . The implementation is numerically stable because by construction, $0 \leq F_{c t} \leq 1$ and $0 \leq G\left(\mathbf{z}_{t} ; \mu_{c s}, \sigma_{c}\right) G\left(t ; \nu_{c s}, \rho_{c}\right) / P_{c t} \leq 1$. For the application in this paper, the iterative optimization method took about 4 iterations to converge; each iteration taking about 5 minutes using unoptimized Matlab code.

\section{Results, Validation, and Discussion}

We obtain real DT images: $128 \times 128 \times 40$ voxels; voxel size $1.7 \times 1.7 \times 3 \mathrm{~mm}$; singleshot spin-echo diffusion-weighted echo-planar imaging; 12 images per subject; 12 isotropically-distributed diffusion-encoding directions $\left(b=1000 \mathrm{~s} / \mathrm{mm}^{2}\right)$. 


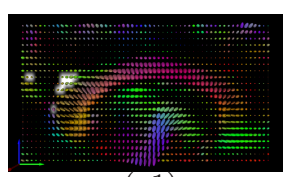

(a1)

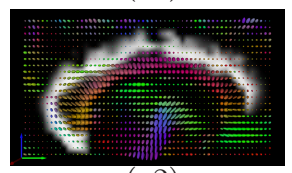

(a2)

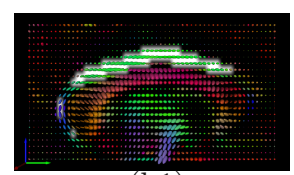

(b1)

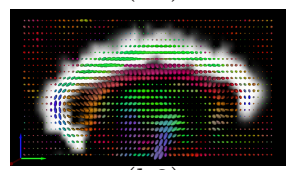

(b2)

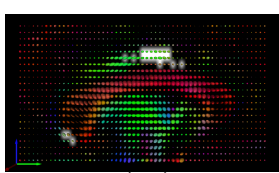

(c1)

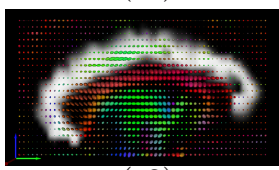

(c2)

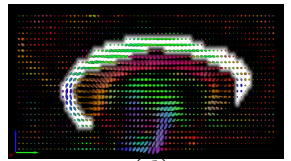

(d)

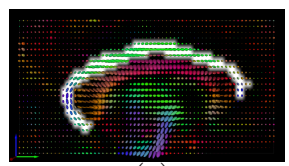

(e)

Fig. 1. The extracted cingulum, seen as the white background in the glyph-based color-coded DT visualization (sagittal slices), using: (a1)-(c1) tractography, and (a2)(c2) proposed fuzzy-segmentation-based approach (white $\Rightarrow$ fuzzy membership value $F_{c t}=1$, black $\Rightarrow F_{c t}=0$, gray $\Rightarrow 0<F_{c t}<1$ ). (d) A manual segmentation (crisp) for the slice in (b1) or (b2) superimposed on the DT image. (e) An initialization (1 slice only; slice in (b1)or (b2)) for the segmentation superimposed on the DT image.

For extracting each cingulum in the brain, we obtained an initial (crisp) segmentation, on just a single sagittal slice of the cingulum, by thresholding tensors that: (i) have high FA (to separate white matter from the rest of the brain) and (ii) are orientated along anterior-posterior or inferior-superior directions. We manually remove voxels in the initialization (one slice) that clearly belonged to other far-off tracts, e.g. the fornix lying inferior to the corpus callosum [3].

Figure1(a)-(c) shows the results of a standard tractography technique for the tract extraction using two regions-of-interest in the superior part of the cingulum. It is clear that tractography fails to extract the cingulum - a thin tract with sharp changes in orientation. On the other hand, Figure1(d)-(f) shows that the proposed fuzzy segmentation approach - that exploits the statistical coherence of tensors in the entire structure-performs significantly better. Figure 2 shows the result, on another real DT image. For validation and quantitative comparison, we obtain two manual (crisp) segmentations by using interactive software to delineate color-coded scalar FA images - the color at each voxel is derived from the orientation of the tensor at that voxel. The Dice overlap metrics (averaged

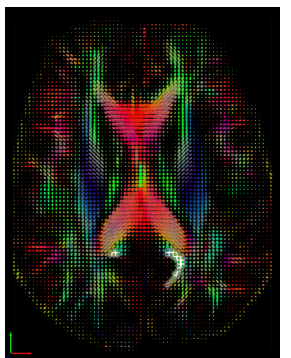

(a)

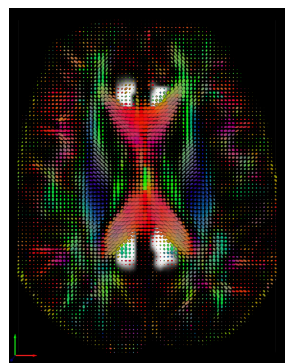

(b)

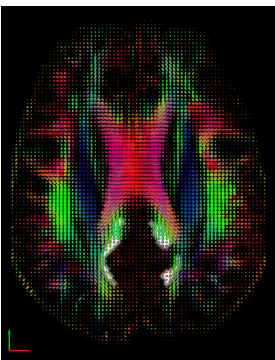

(c)

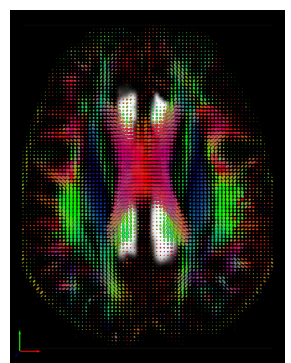

(d)

Fig. 2. The extracted cingulum in axial slices (inferior $\rightarrow$ superior) using: (a),(c) tractography, and (b),(d) proposed fuzzy-segmentation-based approach 


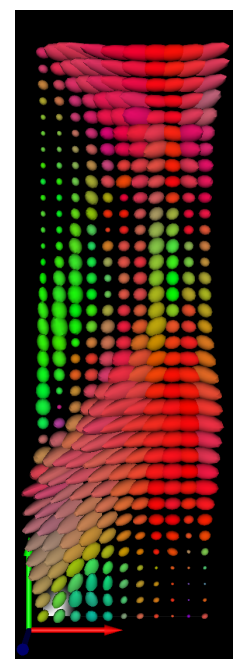

(a)

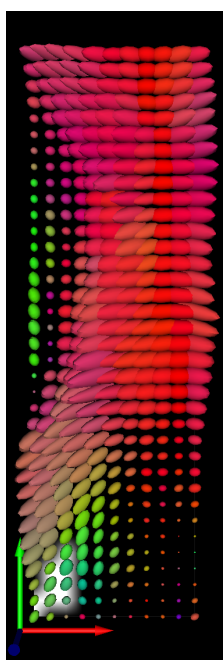

(b)

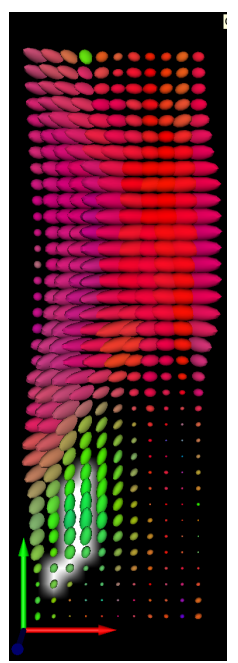

(c)

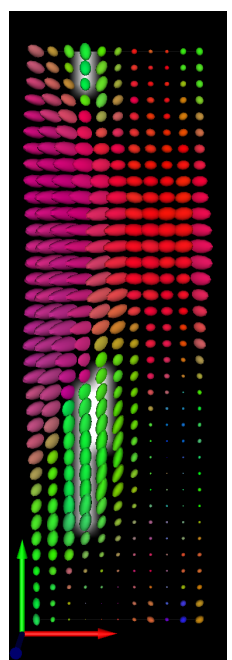

(d)

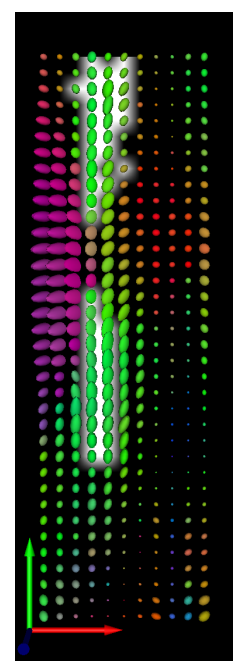

(e)

Fig. 3. Effect of partial voluming on tractography: (a)-(e) show regions near the cingulum/corpus-callosum interface in consecutive axial slices (inferior $\rightarrow$ superior)

over the two manual segmentations) for these two DT images were: (i) 0.63 and 0.60 for the proposed method (after thresholding the fuzzy membership values $F_{c t}$ with a value of 0.5 ) and (ii) 0.32 and 0.33 for tractography.

Figure 3 shows enlarged axial views of the tractography result near the interface of the corpus callosum (red) and the cingulum (green). Significant partial voluming in this region, worsened by the low inter-axial-slice resolution, leads to a change in the orientation of the cingulum tensors. Thus, many cingulum tensors are no longer aligned with the direction of the cingulum tract, but rather have deviated orientations (brown) towards the orientation of the corpus callosum. Such phenomena cause fiber tracking, which uses thresholds on FA and curvature, to either: (i) wander far away from the desired cingulum tract, e.g. Figure 2(a)-(b), (loose thresholds) or (ii) terminate prematurely (conservative thresholds). In addition to this inherent trade-off, tractography suffers because it relies solely on the orientation of the current tensor and ignores the information in the tensors that were previously tracked. The proposed method-by optimally exploiting the information contained in all the tensor values, locations, and partial-volumed voxels in a unified framework - extracts the cingulum with significantly higher accuracy. Future work includes better quantification of the improvements obtained with the proposed method.

\section{Acknowledgments}

The authors gratefully acknowledge NIH support of this work via grants HD042974, HD046159, NS045839, and EB06266. 


\section{References}

1. Lenglet, C., Rousson, M., Deriche, R., Faugeras, O., Lehericy, S., Ugurbil, K.: A Riemannian approach to diffusion tensor image segmentation. In: Proc. Info. Proc. Med. Imag, pp. 591-602 (2005)

2. Wang, Z., Vemuri, B.: DTI segmentation using an information theoretic tensor dissimilarity measure. IEEE Trans. Med. Imag. 24(10), 1267-1277 (2005)

3. Concha, L., Gross, D., Beulieu, C.: Diffusion tensor tractography of the limbic system. Amer. J. Neuroradiology 26, 2267-2274 (2005)

4. Jiang, G., Zang, Z., Xie, W., Guo, X.: Asymmetry analysis of cingulum based on scale-invariant parameterization by diffusion tensor imaging. Human Brain Map 24, 92-98 (2005)

5. Ziyan, U., Tuch, D., Westin, C.F.: Segmentation of thalamic nuclei from DTI using spectral clustering. In: Proc. Med. Image Comput. and Comp. Assisted Intervention, pp. 807-814 (2006)

6. Franklin, T., Acton, P., Maldjian, J., Gray, J., Croft, J., Dackis, C., O'Brien, C., Childress, A.: Decreased gray matter concentration in the insular, orbitofrontal, cingulate, and temporal cortices of cocaine patients. Biol. Psychiatry 51(2), 134 $142(2002)$

7. Kinoshita, M., Yamada, K., Hashimoto, N., Kato, A., Izumoto, S., Baba, T., Maruno, M., Nishimura, T., Yoshimine, T.: Fiber tracking does not accurately estimate size of fiber bundles in pathological conditions: initial neurosurgical experience using neuronavigation and subcortical white matter stimulation. NeuroImage 25(2), 424-429 (2005)

8. Zhuang, X., Huang, Y., Palaniappan, K., Zhao, Y.: Gaussian mixture density modeling, decomposition, and applications. IEEE Trans. Image Proc. 5(9), 1293-1302 (1996)

9. Leemput, K.V., Maes, F., Vandermeulen, D., Seutens, P.: Automated model-based tissue classification of MR images of the brain. IEEE Tr. Med. Imaging 18, 897-908 (1999)

10. Pham, D., Prince, J.: An adaptive fuzzy segmentation algorithm for threedimensional magnetic resonance images. In: Proc. Info. Proc. Med. Imag, 140-153 (1999)

11. Kim, J., Fisher, J.W., Yezzi, A.J., Cetin, M., Willsky, A.S.: A nonparametric statistical method for image segmentation using information theory and curve evolution. IEEE Trans. Image Processing 14(10), 1486-1502 (2005)

12. Awate, S.P., Tasdizen, T., Foster, N.L., Whitaker, R.T.: Adaptive, nonparametric Markov modeling for unsupervised, MRI brain-tissue classification. Medical Image Analysis 10(5), 726-739 (2006)

13. Fletcher, P.T., Joshi, S.C.: Principal geodesic analysis on symmetric spaces: Statistics of diffusion tensors. In: ECCV Workshops CVAMIA and MMBIA, pp. 87-98 (2004)

14. Arsigny, V., Fillard, P., Pennec, X., Ayache, N.: Geometric means in a novel vector space structure on symmetric positive-definite matrices. SIAM J. Matrix Analysis and Applications (in press)

15. Pennec, X., Fillard, P., Ayache, N.: A Riemannian framework for tensor computing. Int. J. Comput. Vision 66(1), 41-66 (2006)

16. Awate, S.P., Gee, J.C.: A fuzzy, nonparametric segmentation framework for DTI and MRI analysis. In: Proc. Info. Proc. in Med. Imag (IPMI) (to appear, 2007)

17. Chow, Y., Geman, S., Wu, L.: Consistant cross-validated density estimation. Annals of Statistics 11(1), 25-38 (1983) 